\title{
Shape anisotropy and magnetic domain structures in striped monolayers
}

\author{
Paolo Politi ${ }^{1}$ and Maria Gloria Pini²
}

\author{
August 14, 2018 \\ ${ }^{1}$ Dipartimento di Fisica dell'Università degli Studi di Firenze and Sezione INFM, L.go E. \\ Fermi 2, I-50125 Firenze, Italy \\ ${ }^{2}$ Istituto di Elettronica Quantistica, Consiglio Nazionale delle Ricerche, Via Panciatichi \\ 56/30, I-50127 Firenze, Italy
}

\begin{abstract}
We study the effect of dipolar interactions on a magnetic striped monolayer with a microscopic unit cell of square symmetry, and of size $\left(N_{x} \times N_{y}\right)$ spins. Even if the aspect ratio $r=N_{x} / N_{y}$ is very large, an in-plane shape anisotropy is always negligible, except if $N_{y}$ is fairly small $\left(N_{y}<40\right)$. In-plane domains are not possible, except for values of the dipolar coupling larger than the domain wall energy.
\end{abstract}

\section{Introduction}

An important and well known feature of magnetic dipole-dipole interactions is that, in spite of their weakness with respect to exchange coupling, they play an important role in magnetic systems [1]. In fact, the long range character of the magnetostatic interactions is relevant for determining both the ground state of the system [2] and the excitation spectrum [3]; perhaps, the most striking consequence of dipolar interaction is the breaking of a bulk sample in several magnetic domains.

The peculiarity of dipolar interactions in three dimensions is elucidated by the fact that a shape anisotropy is always present, independently of the size of the sample: as a consequence of this, it has no meaning to speak about an "infinite sample" without specifing its limiting shape. For example, for the slab geometry (see Fig. 1b), the demagnetizing factors are 0 in the $x, y$ directions and 1 in the $z$ one, perpendicular to the slab. Thus, the in-plane collinear state has a lower energy than the perpendicular collinear one, whose surface energy density turns out to be proportional to the thickness of the slab, and therefore the shape anisotropy per spin of a slab is a constant and doesn't depend on its thickness $N_{z}$ !

In genuine two dimensional magnetic systems, like magnetic films of atomic thickness ( Fe, $\mathrm{Co}, \mathrm{Ni}, \mathrm{Gd}$ ) grown on nonmagnetic substrates $(\mathrm{Au}, \mathrm{Ag}, \mathrm{Cu}, \ldots$ ), the situation is quite different: the shape anisotropy due to magnetic dipole-dipole interactions favours an inplane magnetization without establishing any preferential direction within the film [4, 5], and the magnetization direction of a collinear configuration is determined by the competition with possible easy-axis anisotropies generated by the breaking of the translational invariance in the growth direction and which may favour a perpendicular state. For in-plane 
magnetization, a monodomain configuration is energetically favoured; for perpendicular magnetization, the system prefers to break into domains [6, 7], however weak the dipolar interaction may be.

Recently, two-dimensional mesoscopic structures [8] have attracted attention for their potential technological applications. They are characterized (see Fig. 1a) by a "small" lateral dimension in one direction $\left(N_{y} \ll N_{x}\right.$ : magnetic wires) or in both directions (magnetic dots). Such structures will be called striped monolayers in the following, and $N_{x}, N_{y}$ will be allowed to assume any value.

The aim of the present paper is to elucidate the role played by magnetic dipole-dipole interactions in such systems, provided that the magnetization lies in the plane of the stripe and the microscopic unit cell has square symmetry, and to ascertain whether the results valid for a slab apply analogously, namely: (i) the presence of a strong shape anisotropy favouring the $x$ direction if $N_{x} \gg N_{y}$, and (ii) the existence of domains, if some in-plane magnetocrystalline anisotropy forces the magnetization along the $y$ direction. As we will show, neither of the previous points holds. In contrast, we find that (i) even for a stripe with an infinite aspect ratio $\left(N_{x} / N_{y} \rightarrow \infty\right)$, the shape anisotropy per spin vanishes upon increasing $N_{y}$, and (ii) in-plane domain structures can appear only for very large values of the dipolar interaction $\Omega$.

\section{Shape anisotropy of a striped monolayer}

Let us start by writing down the dipolar energy for an in-plane collinear ground state:

$$
E_{d i p}=\frac{\Omega}{2} \sum_{\vec{n}, \vec{m}} \frac{1}{r_{n m}^{3}}\left[1-3 \frac{\left(\vec{S} \cdot \vec{r}_{n m}\right)^{2}}{r_{n m}^{2}}\right]
$$

where the distance $r_{n m}=|\vec{n}-\vec{m}|$ (with $\vec{n} \neq \vec{m}$ ) is measured in units of the square lattice constant.

The shape anisotropy is determined solely by the anisotropic part of $E_{\text {dip }}$ (the second term in square brackets), which becomes, if $\vec{S}=(\cos \theta, \sin \theta)$ and up to a constant:

$$
E_{d i p}=-3 \Omega \cos ^{2} \theta \cdot \frac{1}{2} \sum_{\vec{n}, \vec{m}} \frac{\left(x_{n m}^{2}-y_{n m}^{2}\right)}{r_{n m}^{5}} \equiv-3 \Omega \cos ^{2} \theta \cdot \mathcal{S},
$$

where the "cross" term of the form $\sum_{\vec{n}, \vec{m}}\left(x_{n m} y_{n m} / r_{n m}^{5}\right)$ vanishes if the system has at least one symmetry axis (a rectangular monolayer has two).

The previous expression vanishes for an infinite two dimensional monolayer, or for any square-shaped system $\left(N_{x}=N_{y}\right)$, provided that the symmetry of the microscopic unit cell is square. For a rectangle with $N_{x}>N_{y}$, a couple of points $(\vec{n}, \vec{m})$ will generally correspond to a value $\left|x_{n m}\right|>\left|y_{n m}\right|$, so that the summation $\mathcal{S}$ will have a strictly positive value, $E_{d i p}$ will be minimal for $\theta=0$ and the magnetization will be oriented along the $\hat{x}$ axis.

The relevant quantity is the dipolar energy per spin: $\epsilon_{d i p}=E_{d i p} / N_{x} N_{y}$, and we are interested in analyzing the dependence of $\epsilon_{d i p}$ on $N_{x}$ and $N_{y}$. For $N_{y}=1$, in the limit of an infinite chain of spins, it is immediately found that

$$
\epsilon_{\text {dip }}\left(N_{x}=\infty, N_{y}=1\right)=-3 \Omega \cos ^{2} \theta \cdot \frac{1}{2} \sum_{n \neq 0} \frac{1}{n^{3}} \equiv-3 \Omega \cos ^{2} \theta \cdot \zeta(3),
$$


where $\zeta(3)=1.2021$ is the Riemann's zeta function 9].

The competition between the dipolar contribution $(3 \zeta(3) \Omega)$ and possible in-plane anisotropies favouring the $\hat{y}$ axis, determines the actual direction of the magnetization. In the following, we will evaluate $\epsilon_{d i p}$ in a continuum approximation, for any value of $N_{x}, N_{y}$.

The exact definition of $\mathcal{S}$ is:

$$
\mathcal{S} \equiv \frac{1}{2} \sum_{l, l^{\prime}=1}^{N_{x}} \sum_{m, m^{\prime}=1}^{N_{y}} \frac{\left(l-l^{\prime}\right)^{2}-\left(m-m^{\prime}\right)^{2}}{\left[\left(l-l^{\prime}\right)^{2}+\left(m-m^{\prime}\right)^{2}\right]^{5 / 2}} \quad \text { with } \quad(l, m) \neq\left(l^{\prime}, m^{\prime}\right) .
$$

It is useful to introduce the new integer variables: $x=\left(l-l^{\prime}\right)$, with $-\left(N_{x}-1\right) \leq x \leq$ $\left(N_{x}-1\right)$ and $y=\left(m-m^{\prime}\right)$, with $-\left(N_{y}-1\right) \leq y \leq\left(N_{y}-1\right)$. $\mathcal{S}$ now writes as a sum on solely two indices:

$$
\mathcal{S}=\frac{1}{2} \sum_{x=-\left(N_{x}-1\right)}^{N_{x}-1} \sum_{y=-\left(N_{y}-1\right)}^{N_{y}-1}\left(N_{x}-|x|\right)\left(N_{y}-|y|\right) \frac{x^{2}-y^{2}}{\left(x^{2}+y^{2}\right)^{5 / 2}}
$$

At this point we exploit the fact that $\mathcal{S} \equiv 0$ if $N_{x}=N_{y}$. Therefore, we will write $N_{x}=N_{y}+\left(N_{x}-N_{y}\right)$ :

$$
\begin{aligned}
\mathcal{S} & =\frac{1}{2} \sum_{|x|,|y| \leq\left(N_{y}-1\right)}\left(N_{y}-|x|\right)\left(N_{y}-|y|\right) \frac{x^{2}-y^{2}}{\left(x^{2}+y^{2}\right)^{5 / 2}} \\
& +\frac{1}{2} \sum_{|x|>\left(N_{y}-1\right)} \sum_{|y| \leq\left(N_{y}-1\right)}\left(N_{x}-|x|\right)\left(N_{y}-|y|\right) \frac{x^{2}-y^{2}}{\left(x^{2}+y^{2}\right)^{5 / 2}} \\
& +\frac{1}{2}\left(N_{x}-N_{y}\right) \sum_{|x|,|y| \leq\left(N_{y}-1\right)}\left(N_{y}-|y|\right) \frac{x^{2}-y^{2}}{\left(x^{2}+y^{2}\right)^{5 / 2}}
\end{aligned}
$$

The first sum vanishes, as seen by interchanging the two dumb indices $(x, y)$; in the third sum, for the same reason we can get rid of the quantity proportional to $\left(N_{x}-N_{y}\right) N_{y}\left(x^{2}-\right.$ $\left.y^{2}\right) /\left(x^{2}+y^{2}\right)^{5 / 2}$, whilst in the other one the term $y=0$ does not contribute. Finally, by using the parity of the addenda, we obtain:

$$
\begin{aligned}
\mathcal{S} & =\sum_{x=N_{y}}^{N_{x}-1} \sum_{y=-\left(N_{y}-1\right)}^{N_{y}-1}\left(N_{x}-x\right)\left(N_{y}-|y|\right) \frac{x^{2}-y^{2}}{\left(x^{2}+y^{2}\right)^{5 / 2}} \\
& -\left(N_{x}-N_{y}\right) \sum_{y=1}^{N_{y}-1} \sum_{x=-\left(N_{y}-1\right)}^{N_{y}-1} y \frac{x^{2}-y^{2}}{\left(x^{2}+y^{2}\right)^{5 / 2}}
\end{aligned}
$$

It is noteworthy that the previous expression is exact: its use for a numerical calculation of $\mathcal{S}$ requires the evaluation of $2 N_{x} N_{y}$ terms, whilst Eq. (14) demanded $N_{x}^{2} N_{y}^{2}$ terms!

The evaluation of $\mathcal{S}$ in the continuum approximation $\left(\sum_{x, y} \rightarrow \iint d x d y\right)$ is performed in the Appendix A. Here we will discuss the results. First of all, let us consider the case of an infinite aspect ratio $\left(r=N_{x} / N_{y}=\infty\right.$ with $N_{y}$ finite). The quantity $\mathcal{S}$ per spin is (see Eq. (26)):

$$
\frac{\mathcal{S}}{N_{x} N_{y}}=\frac{2}{3 N_{y}} \ln N_{y}+\frac{c_{1}}{N_{y}}
$$

An important feature of the previous expression immediately comes out: the shape anisotropy (per spin) vanishes upon increasing $N_{y}$, even if $N_{x} / N_{y}=\infty$ ! This means that 
the shape anisotropy of an infinite stripe $\left(N_{x}=\infty\right)$ becomes rapidly negligible, as $N_{y}$ increases.

The numerical value of $c_{1}$ cannot be determined by our "zero-order" continuum approximation; in fact, the first-order correction, given by the term in square brackets of the Euler-Maclaurin summation formula (see Ref. [9]):

$$
\sum_{x=a}^{b} f(x)=\int_{a}^{b} d x f(x)+\frac{1}{2}[f(a)+f(b)]+\ldots
$$

contributes just to the term of order $\left(1 / N_{y}\right)$ in (7). Since the leading term $\left(\approx\left(1 / N_{y}\right) \ln N_{y}\right)$ dominates only logarithmically, the constant cannot be neglected. In Eq. (7), $c_{1}$ plays the role of the anisotropy for a single line of spins:

$$
c_{1}=\left.\frac{\mathcal{S}}{N_{x} N_{y}}\right|_{N_{y}=1}=\sum_{l=1}^{\infty} \frac{1}{l^{3}}=\zeta(3) .
$$

So, we will rewrite (7) in the form:

$$
\frac{\mathcal{S}}{N_{x} N_{y}}=\frac{2}{3 N_{y}} \ln N_{y}+\frac{\zeta(3)}{N_{y}} .
$$

In the limit $N_{x}=\infty$, the numerical calculation of $\mathcal{S} / N_{x}$ can be made much more efficient by means of the Ewald's summation technique [10], which allows to rewrite $\mathcal{S} / N_{x}$ as an exponentially converging sum. This is done in Appendix B. We are therefore able to compare (see Fig. 2) the exact numerical result with the analytic approximation (10). Even our "zero-order" continuum approximation gives a fairly good approximation.

The following considerations are meant to corroborate further on the previous results. If $N_{x}=\infty$, we can exploit the translational invariance in the $\hat{x}$ direction to write down:

$$
\begin{aligned}
\frac{\mathcal{S}}{N_{x}} & =\frac{1}{2} \sum_{m, m^{\prime}=1}^{N_{y}} \sum_{l=-\infty}^{\infty} \frac{l^{2}-\left(m-m^{\prime}\right)^{2}}{\left[l^{2}+\left(m-m^{\prime}\right)^{2}\right]^{5 / 2}} \equiv \frac{1}{2} \sum_{m, m^{\prime}=1}^{N_{y}} S\left(m-m^{\prime}\right) \\
& =\frac{1}{2} N_{y} S(0)+\sum_{c=1}^{N_{y}-1}\left(N_{y}-c\right) S(c)
\end{aligned}
$$

$S(c)$ represents the "interaction" per unit length of two lines at distance $c$, whilst $S(0) / 2$ is the self-interaction of a line. In the limit where $N_{y}$ can be treated as a continuous variable:

$$
\begin{aligned}
\frac{\partial}{\partial N_{y}}\left(\frac{\mathcal{S}}{N_{x}}\right) & =\frac{S(0)}{2}+\sum_{c=1}^{N_{y}} S(c) \\
\frac{\partial^{2}}{\partial N_{y}^{2}}\left(\frac{\mathcal{S}}{N_{x}}\right) & =S\left(N_{y}\right)
\end{aligned}
$$

If the continuum approximation is applied also to the $\hat{x}$ direction, for $c \neq 0$ we will have:

$$
S(c)=\int_{-\infty}^{+\infty} d x \frac{x^{2}-c^{2}}{\left(x^{2}+c^{2}\right)^{5 / 2}}=-\frac{2}{3 c^{2}}
$$

So,

$$
\frac{\partial^{2}}{\partial N_{y}^{2}}\left(\frac{\mathcal{S}}{N_{x}}\right)=-\frac{2}{3 N_{y}^{2}}
$$


which gives

$$
\frac{\mathcal{S}}{N_{x}}=\frac{2}{3} \ln N_{y}+c_{0} N_{y}+c_{1}
$$

This expression would disagree with (7), if $c_{0}$ were not zero. It is zero, indeed. In fact:

$$
c_{0}=\lim _{N_{y} \rightarrow \infty} \frac{\partial}{\partial N_{y}}\left(\frac{\mathcal{S}}{N_{x}}\right)=\frac{S(0)}{2}+\sum_{c=1}^{\infty} S(c)
$$

and by writing down $\left(\frac{\mathcal{S}}{N_{x} N_{y}}\right)$ for a completely translationally invariant two dimensional system, we obtain:

$$
\begin{aligned}
\frac{\mathcal{S}}{N_{x} N_{y}} & =\frac{1}{2} \sum_{(m, l) \neq(0,0)} \frac{l^{2}-m^{2}}{\left(l^{2}+m^{2}\right)^{5 / 2}}=0 \\
& =\frac{S(0)}{2}+\sum_{m=1}^{\infty} S(m)=c_{0}
\end{aligned}
$$

Therefore $c_{0}$ vanishes and Eq. (12) reduces to Eq. (7).

After having discussed the shape anisotropy of an infinite stripe $\left(N_{x}=\infty\right)$, upon increasing its "thickness" $N_{y}$, now let us analyze the dependence of $\mathcal{S} / N_{x} N_{y}$ on the aspect ratio $r$. By handling the expression for $\frac{\mathcal{S}}{N_{x} N_{y}}$ given in Appendix and by using the "boundary" condition $\frac{\mathcal{S}}{N_{x} N_{y}}\left(N_{x}=\infty, N_{y}=1\right)=\zeta(3)$, we obtain:

$$
\frac{\mathcal{S}}{N_{x} N_{y}}=\left(1-\frac{1}{r}\right)\left[\frac{2}{3 N_{y}} \ln N_{y}+\frac{\zeta(3)}{N_{y}} F(r)\right],
$$

where $F(\infty)=1$ and $F(1) \approx 1$ : the actual value of $F(1)$ is not really relevant, because the shape anisotropy vanishes in the limit $r=1$.

In Fig. 3 we compare the previous expression (as a function of $r$, keeping fixed the value of $N_{y}=40$ ) with the exact numerical result, obtained by exploiting Eq. (6). The behavior is well reproduced by the analytical expression.

\section{Domain structures in a striped monolayer}

Now, let us turn to the study of domain structures in striped monolayers. Our purpose is to check if - and when - the appearance of magnetic domains is energetically favoured. We will consider a striped monolayer which is infinite in the $\hat{x}$ direction $\left(N_{x}=\infty\right)$ and we ask for which values of the parameters, the creation of a domain wall along the $\hat{y}$ axis makes the system gain energy with respect to the collinear FM state.

We will consider two cases, according to the direction of the magnetization: perpendicular to the striped monolayer, and in the plane, along the "hard" direction $(\hat{y})$. We consider just these two possibilities, because a striped monolayer has a "double" shape anisotropy: it has an easy-plane effect (as in a film, or in an infinite monolayer), but in the plane, it has also an easy-axis effect, along the $\hat{x}$ direction, which has been discussed in detail in the previous section. If the easy-plane effect is overcome by some anisotropies $\left(K_{\perp}\right)$ oriented along $\hat{z}$, the film will break into domains. So, our first purpose (I) will be to study the finite size effects on this domain structure. 
Conversely, if the easy-plane effect of $E_{d i p}$ prevails, the magnetization will be oriented along $\hat{x}$, or -in presence of a sufficiently strong anisotropy $K_{\|}$favouring the $\hat{y}$ axis- along the smaller size of the stripe. In analogy with (I), we will expect that the striped monolayer breaks into in-plane domains. We will analyze (II) this possibility, by showing that domains don't appear, except for very large values of the dipolar coupling.

What we have to do -in cases (I) and (II) - is to create a single domain wall along the $\hat{y}$ axis, and to compare the domain wall energy, with the dipolar energy gain. In the following, $w$ will indicate the domain wall size. For an evaluation of the dipolar term, in the Heisenberg model it will be sufficient to consider Ising-like spins, with domains separated by an empty region of size $w$ 11].

In both cases (out-of-plane and in-plane domains) the domain wall energy per unit length in the $\hat{y}$ direction, will be written as $E_{d w}$ (for the Ising model: $E_{d w}=2 J$, where $J$ is the exchange coupling constant, and for the Heisenberg model: $E_{d w}=2 \sqrt{J K}$, where $K=K_{\perp}$ in case (I) and $K=K_{\|}$in case (II)).

I: out-of-plane domains. Because of the finite extension of the striped monolayer, the translational invariance in the $\hat{y}$ direction is lost and the dipolar interaction between a given spin and the spins of a neighbouring domain depends on the $\hat{y}$ coordinate of the spin. However, for the evaluation of the order of magnitude of the dipolar energy gain (per unit length), we can use the following approximate formula is nothing but the dipolar interaction between two spins located in $\left(x^{\prime}, 0\right)$ and $(x, y)$, and pointing along $\pm \hat{z}$ :

$$
\begin{aligned}
\Delta E_{d i p}\left(N_{y}\right) & \approx-2 \Omega \int_{-\infty}^{0} d x^{\prime} \int_{0}^{N_{y}} d y \int_{w}^{\infty} \frac{d x}{\left[\left(x-x^{\prime}\right)^{2}+y^{2}\right]^{3 / 2}} \\
& \approx-2 \Omega\left[\ln \left(\frac{N_{y}+\sqrt{N_{y}^{2}+w^{2}}}{w}\right)-\frac{\sqrt{N_{y}^{2}+w^{2}}-w}{N_{y}}\right]
\end{aligned}
$$

In the limit $N_{y} \gg w, \Delta E_{d i p} \approx-2 \Omega \ln \left(2 N_{y} / w\right)$. The $\mathrm{FM} \perp$ state will be destabilized if $\left|\Delta E_{d i p}\right|>E_{d w}$, or

$$
N_{y}>\frac{w}{2} \exp \left(\frac{E_{d w}}{2 \Omega}\right)
$$

The explanation of this result is straightforward: out-of-plane domains will appear only if the lateral dimensions of the striped monolayer are larger than the size $L$ that domains would have in an infinite monolayer. In fact, the right-hand side of Eq. (16) is exactly the typical size of a domain in an infinite monolayer [12, 13.

II: in-plane domains, magnetized along $\pm \hat{y}$. In this case, $\Delta E_{\text {dip }}$ writes:

$$
\begin{aligned}
\Delta E_{d i p} & \approx-2 \Omega \int_{-\infty}^{0} d x^{\prime} \int_{0}^{N_{y}} d y \int_{w}^{\infty} d x\left[\frac{1}{\left[\left(x-x^{\prime}\right)^{2}+y^{2}\right]^{3 / 2}}-\frac{3 y^{2}}{\left[\left(x-x^{\prime}\right)^{2}+y^{2}\right]^{5 / 2}}\right] \\
& \approx-2 \Omega \frac{\sqrt{N_{y}^{2}+w^{2}}-w}{N_{y}},
\end{aligned}
$$

where the dipolar interaction $-\Omega\left\{1 /\left[\left(x-x^{\prime}\right)^{2}+y^{2}\right]^{3 / 2}-3 y^{2} /\left[\left(x-x^{\prime}\right)^{2}+y^{2}\right]^{5 / 2}\right\}$ between two spins located in $\left(x^{\prime}, 0\right)$ and $(x, y)$ now has a contribution from the anisotropic part (the second term of Eq. (11)).

\footnotetext{
${ }^{1}$ Indeed, it is possible to show that the dipolar energy gain is in between $\Delta E_{d i p}\left(N_{y}\right)$ and $2 \Delta E_{d i p}\left(N_{y} / 2\right)$. Since $\Delta E_{\text {dip }}$ depends only logarithmically on $N_{y}$, the correction is not important.
} 
In the limit $N_{y} \gg w$, Eq. (17) simply writes: $\Delta E_{\text {dip }} \approx-2 \Omega$. So, the dipolar energy gain does not increase with $N_{y}$ and therefore the condition for the appearance of in-plane domains $\left(\left|\Delta E_{d i p}\right|>E_{d w}\right)$ can be fulfilled only if $\Omega \approx E_{d w}$, i.e. a rather large value.

\section{Conclusions}

Bulk systems differ substantially from two dimensional ones, because shape effects are relevant in three dimensions, but in-plane shape effects are negligible for stripes. More precisely, if the magnetization lies in the plane, its direction is mainly determined by existing magnetocrystalline anisotropies, because dipolar shape effects rapidly vanish when the sizes of the stripe increase, even if the aspect ratio goes to infinity. In particular, we have shown that the shape anisotropy per spin vanishes as $\frac{\ln N_{y}}{N_{y}}$ upon increasing the size $N_{y}$, so that, e.g. for $N_{y}=40$ (and $N_{x}=\infty$ ) it is reduced by a factor larger than 10 with respect to a single chain of spins $\left(N_{y}=1\right)$.

The extreme weakness of shape effects in stripes has a further consequence on the existence of in-plane domains: in fact, if the magnetization is forced in the $\hat{y}$ direction (the "hard" direction with respect to the shape anisotropy), in-plane domains with the magnetization alternately directed along $\pm \hat{y}$ appear only if $\Omega$ is fairly large.

We remark that our analysis has assumed that the striped magnetic monolayer considered has a microscopic unit cell of square symmetry, so the theory is directly applicable to epitaxial monolayers grown on (100) substrates. In the case of the other high symmetry orientation - the (111) one - the overlayer has a triangular symmetry and no in-plane anisotropy is induced by the dipolar interaction, in the limit of an infinite monolayer. For striped monolayers, our previous treatment should be relevant. Conversely, for (110) substrates the microscopic unit cell has a rectangular symmetry, which induces a further (and possibly competitive) anisotropy in addition to the shape anisotropy.

Acknowledgements - We acknowledge Danilo Pescia for having introduced us to the problem of the striped monolayers.

\section{A Evaluation of $\mathcal{S}$ in the continuum approximation}

In the continuum approximation, Eq. (6) for $\mathcal{S}$ rewrites:

$$
\mathcal{S}=2 I_{1}-2\left(N_{x}-N_{y}\right) I_{2},
$$

where:

$$
\begin{aligned}
I_{1} & =\int_{N_{y}}^{N_{x}-1} d x \int_{0}^{N_{y}-1} d y\left(N_{x} N_{y}+x y-N_{x} y-N_{y} x\right) \mathcal{A}(x, y) \\
I_{2} & =\int_{0}^{N_{y}-1} d x \int_{1}^{N_{y}-1} d y y \mathcal{A}(x, y) \\
\mathcal{A}(x, y) & =\frac{x^{2}-y^{2}}{\left(x^{2}+y^{2}\right)^{5 / 2}}
\end{aligned}
$$


The following integrals are easily calculated [14]:

$$
\begin{aligned}
A\left(x_{1}, x_{2}, y_{1}, y_{2}\right)= & \int_{x_{1}}^{x_{2}} d x \int_{y_{1}}^{y_{2}} d y \mathcal{A}(x, y) \\
= & \frac{y_{1}^{2}-x_{2}^{2}}{3 y_{1} x_{2} \sqrt{y_{1}^{2}+x_{2}^{2}}}+\frac{x_{2}^{2}-y_{2}^{2}}{3 x_{2} y_{2} \sqrt{x_{2}^{2}+y_{2}^{2}}}+\left[\left(x_{1}, y_{1}\right) \leftrightarrow\left(x_{2}, y_{2}\right)\right] \\
B\left(x_{1}, x_{2}, y_{1}, y_{2}\right) & =\int_{x_{1}}^{x_{2}} d x \int_{y_{1}}^{y_{2}} d y x y \mathcal{A}(x, y) \\
& =\frac{y_{1}^{2}-x_{2}^{2}}{3 \sqrt{y_{1}^{2}+x_{2}^{2}}}+\frac{x_{2}^{2}-y_{2}^{2}}{3 \sqrt{x_{2}^{2}+y_{2}^{2}}}+\left[\left(x_{1}, y_{1}\right) \leftrightarrow\left(x_{2}, y_{2}\right)\right] \\
C\left(x_{1}, x_{2}, y_{1}, y_{2}\right) & =\int_{x_{1}}^{x_{2}} d x \int_{y_{1}}^{y_{2}} d y \mathcal{A}(x, y) \\
& =\frac{1}{3} \ln \left[\frac{y_{1}+\sqrt{y_{1}^{2}+x_{2}^{2}}}{y_{1}+\sqrt{y_{1}^{2}+x_{1}^{2}}}\right]+\frac{2 y_{1}}{3 \sqrt{y_{1}^{2}+x_{2}^{2}}}-\frac{2 y_{1}}{3 \sqrt{y_{1}^{2}+x_{1}^{2}}} \\
& +\left[\left(x_{1}, y_{1}\right) \leftrightarrow\left(x_{2}, y_{2}\right)\right] \\
D\left(x_{1}, x_{2}, y_{1}, y_{2}\right) & =\int_{x_{1}}^{x_{2}} d x \int_{y_{1}}^{y_{2}} d y y \mathcal{A}(x, y)=-C\left(y_{1}, y_{2}, x_{1}, x_{2}\right)
\end{aligned}
$$

In the previous expressions, $\left[\left(x_{1}, y_{1}\right) \leftrightarrow\left(x_{2}, y_{2}\right)\right]$ means that we have to interchange $x_{1}$ with $x_{2}$ and $y_{1}$ with $y_{2}$.

After some lengthy, but easy, calculations it is found that

$$
I_{1}=N_{x} N_{y} A_{1}+B_{1}-N_{x} D_{1}-N_{y} C_{1}
$$

where

$$
\begin{aligned}
& A_{1}=\frac{N_{x}^{2}-N_{y}^{2}}{3 N_{x} N_{y} \sqrt{N_{x}^{2}+N_{y}^{2}}}-\frac{\sqrt{2}}{3 N_{y}^{2}} \\
& B_{1}=\frac{N_{x}^{2}-N_{y}^{2}}{3 \sqrt{N_{x}^{2}+N_{y}^{2}}}-\frac{\sqrt{2}}{3}+\frac{N_{y}-N_{x}}{3} \\
& C_{1}=\frac{1}{3} \ln \left[\frac{N_{x}(1+\sqrt{2})}{N_{y}+\sqrt{N_{x}^{2}+N_{y}^{2}}}\right]+\frac{\sqrt{2}}{3}-\frac{2 N_{y}}{3 \sqrt{N_{x}^{2}+N_{y}^{2}}} \\
& D_{1}=\frac{1}{3} \ln \left[\frac{N_{x}+\sqrt{\left.N_{x}^{2}+N_{y}^{2}\right)}}{N_{x}(1+\sqrt{2})}\right]-\frac{\sqrt{2}}{3}+\frac{2 N_{x}}{3 \sqrt{N_{x}^{2}+N_{y}^{2}}}
\end{aligned}
$$

and

$$
I_{2}=\frac{1}{3} \ln \left[\frac{1+\sqrt{2}}{2 N_{y}}\right]-\frac{2-\sqrt{2}}{3}
$$

As a function of the previous quantities, the shape anisotropy per spin writes:

$$
\frac{\mathcal{S}}{2 N_{x} N_{y}}=\frac{I_{1}}{N_{x} N_{y}}-\frac{N_{x}-N_{y}}{N_{x} N_{y}} I_{2}=A_{1}+\frac{B_{1}}{r N_{y}^{2}}-\frac{D_{1}}{N_{y}}-\frac{C_{1}}{r N_{y}}-\left(1-\frac{1}{r}\right) \frac{I_{2}}{N_{y}}
$$


In the limit $N_{x} \rightarrow \infty$ it is immediately found that

$$
\frac{\mathcal{S}}{N_{x} N_{y}}=\frac{2}{3 N_{y}} \ln N_{y}+\mathcal{O}\left(\frac{1}{N_{y}}\right)
$$

Conversely, for any value of $r=N_{x} / N_{y}$ we obtain the following expression:

$$
\frac{\mathcal{S}}{N_{x} N_{y}}=\left(1-\frac{1}{r}\right)\left[\frac{2}{3 N_{y}} \ln N_{y}+\frac{F^{\prime}(r)}{N_{y}}\right]
$$

where $F^{\prime}(r)$ is a function which depends only very weakly on the aspect ratio $r: F^{\prime}(1) \approx$ $F^{\prime}(\infty) \approx 1$. Indeed, the term proportional to $F^{\prime}(r)$ corresponds to the term of order $\left(1 / N_{y}\right)$ in (26): i.e. a term which cannot be determined consistently by the used "zero-

order" continuum approximation. Imposing the "boundary condition" $\left.\frac{\mathcal{S}}{N_{x} N_{y}}\right|_{r=\infty, N_{y}=1}=$ $\zeta(3)$, corresponds to put $F^{\prime}(r)=\zeta(3) F(r)$, with $F(\infty)=1$ and $F(1) \simeq 1$.

\section{B Exact calculation of $\mathcal{S}$ for $N_{x}=\infty$}

Let us consider the case of a stripe with an infinite aspect ratio $r=N_{x} / N_{y}=\infty$, i.e. with $N_{x}=\infty$ and finite $N_{y}$. Since in this limit translation invariance is restored along the $x$ direction, it turns out that the summation in Eq. (田) can be rewritten

$$
\frac{\mathcal{S}}{N_{x}}=\frac{1}{2} \sum_{m, m^{\prime}=1}^{N_{y}} \sum_{l=-\infty}^{\infty} \frac{l^{2}-\left(m-m^{\prime}\right)^{2}}{\left[l^{2}+\left(m-m^{\prime}\right)^{2}\right]^{5 / 2}}=\frac{1}{2} N_{y} S(0)+\sum_{c=1}^{N_{y}-1}\left(N_{y}-c\right) S(c)
$$

where $S(c)$, the interaction per unit length of two lines at a distance $c$, is

$$
S(c)=\sum_{l=-\infty}^{\infty} \frac{l^{2}-c^{2}}{\left(l^{2}+c^{2}\right)^{5 / 2}}=\sum_{l=-\infty}^{\infty} \frac{1}{\left(l^{2}+c^{2}\right)^{3 / 2}}-2 \sum_{l=-\infty}^{\infty} \frac{c^{2}}{\left(l^{2}+c^{2}\right)^{5 / 2}}
$$

The self-interaction of a line, $S(0)$, is readily evaluated in terms of the Riemann's zeta function $\zeta(x)$

$$
S(0)=\sum_{l=-\infty}^{\infty} \frac{1}{|l|^{3}}=2 \sum_{l=1}^{\infty} \frac{1}{l^{3}}=2 \zeta(3)=2 \cdot 1.202057
$$

To evaluate $S(c)$ for $c>0$ we use a method which was developed by Ewald 10 for converting two-dimensional dipole sums to a rapidly converging form. First we take into account the identity [14]:

$$
\frac{1}{\alpha^{\nu}}=\frac{1}{\Gamma(\nu)} \int_{0}^{\infty} d t t^{\nu-1} e^{-\alpha t}
$$

to rewrite $S(c)$ as follows

$$
S(c)=\frac{1}{\Gamma(3 / 2)} \sum_{l=-\infty}^{\infty} \int_{0}^{\infty} d t t^{1 / 2} e^{-\left(l^{2}+c^{2}\right) t}-2 c^{2} \frac{1}{\Gamma(5 / 2)} \sum_{l=-\infty}^{\infty} \int_{0}^{\infty} d t t^{3 / 2} e^{-\left(l^{2}+c^{2}\right) t}
$$

Next we employ the identity

$$
\sum_{l=-\infty}^{\infty} e^{-l^{2} t}=\sqrt{\pi} t^{-1 / 2} \sum_{n=-\infty}^{\infty} e^{-(\pi n)^{2} / t}
$$


so that

$$
S(c)=\sum_{n=-\infty}^{\infty} \int_{0}^{\infty} d t e^{-c^{2} t} e^{-(\pi n)^{2} / t}\left[\frac{\sqrt{\pi}}{\Gamma(3 / 2)}-2 c^{2} \frac{\sqrt{\pi}}{\Gamma(5 / 2)} t\right]
$$

The integrals can be exactly evaluated in terms of the modified Bessel functions $K_{\nu}(x)$ [14:

$$
\int_{0}^{\infty} d t e^{-a t} e^{-b / t} t^{\nu-1}=2\left(\frac{b}{a}\right)^{\nu / 2} K_{\nu}(2 \sqrt{a b}) \quad(a, b>0)
$$

At last we obtain, for $c \geq 1$ :

$$
S(c)=\frac{4 \pi}{c} \sum_{n=-\infty}^{\infty}|n| K_{1}(2 \pi c|n|)-\frac{16 \pi^{2}}{3} \sum_{n=-\infty}^{\infty} n^{2} K_{2}(2 \pi c|n|)
$$

At this point we take into account the $x \rightarrow 0$ expansion [9] $K_{\nu}(x) \simeq \frac{1}{2} \Gamma(\nu)\left(\frac{1}{2} x\right)^{-\nu}$, so that the $n=0$ terms in the summation are found to give the finite contributions

$$
\begin{array}{ll}
\lim _{n \rightarrow 0} & n K_{1}(2 \pi c n)=\frac{1}{2 \pi c} \\
\lim _{n \rightarrow 0} & n^{2} K_{2}(2 \pi c n)=\frac{1}{2 \pi^{2} c^{2}}
\end{array}
$$

and finally we obtain for $c \geq 1$

$$
S(c)=-\frac{2}{3 c^{2}}+\frac{8 \pi}{c} \sum_{n=1}^{\infty} n K_{1}(2 \pi c n)-\frac{32 \pi^{2}}{3} \sum_{n=1}^{\infty} n^{2} K_{2}(2 \pi c n)
$$

The first term on the r.h.s. gives the main contribution, which coincides with the result of the continuum limit. The two $n$-summations converge very rapidly because the Bessel functions present an exponential decay for high values of their argument: $K_{\nu}(x) \simeq \sqrt{\frac{\pi}{2 x}} e^{-x}\left[1+\frac{1}{8 x}\left(4 \nu^{2}-\right.\right.$ 1) $\left.+O\left(\frac{1}{x^{2}}\right)\right]$ for $x \rightarrow \infty$. In practice, excellent convergence is obtained summing about ten terms.

\section{References}

[1] A. Aharoni, Introduction to the theory of ferromagnetism (Clarendon Press, Oxford, 1996).

[2] C. Kittel, Rev. Mod. Phys. 21, 541 (1949).

[3] T. Holstein and H. Primakoff, Phys. Rev. 58, 1098 (1940).

[4] S.V. Maleev, Zh. Eksp. Teor. Fiz. 70, 2374 (1976) [Sov. Phys. JETP 43, 1240 (1996)].

[5] Y. Yafet, J. Kwo and E.M. Gyorgy, Phys. Rev. B 33, 6519 (1986).

[6] R. Allenspach, J. Magn. Magn. Mater. 129, 160 (1994).

[7] P. Politi. Domain structures in ultrathin magnetic films. To appear in Comments Cond. Mat. Phys. (1997). 
[8] These structures are generally fabricated through lithographic and/or etching techniques. See, for example: A.O. Adeyeye, J.A.C. Bland, C. Daboo, D.G. Hasko and H. Ahmed, J. Appl. Phys. 82, 469 (1997).

[9] M. Abramowitz and I.A. Stegun (Eds.), Handbook of Mathematical Functions, National Bureau of Standards (Applied Mathematics Series 55).

[10] P.P. Ewald, Ann. Phys. 54, 57 and 519 (1917); ibidem 64, 253 (1921). See also H. Benson and D.L. Mills, Phys. Rev. 178, 839 (1969).

[11] A.B. Kashuba and V.L. Pokrovsky, Phys. Rev. Lett. 70, 3155 (1993); Phys. Rev. B 48, 10335 (1993).

[12] Y. Yafet and E.M. Gyorgy, Phys. Rev. B 38, 9145 (1988).

[13] R. Czech and J. Villain, J. Phys.: Condens. Matter 1, 619 (1989).

[14] I.S. Gradshteyn and I.M. Ryzhik, Table of Integrals, Series, and Products, Academic Press (New York, 1980). 
Figure 1: (a) Striped monolayer of size $N_{x} \times N_{y}$. (b) Three-dimensional slab of size $N_{x} \times$ $N_{y} \times N_{z}\left(\right.$ with $\left.N_{z} \ll N_{x}, N_{y}\right)$.

Figure 2: Shape anisotropy per spin, $\mathcal{S} /\left(N_{x} N_{y}\right)$, for a striped monolayer with infinite aspect ratio $\left(r=N_{x} / N_{y}=\infty\right)$, as a function of $N_{y}$. Dots: exact numerical results, derived from Eqs. (28) and (37). Line: analytical approximation, Eq. (10).

Figure 3: Shape anisotropy per spin, $\mathcal{S} /\left(N_{x} N_{y}\right)$, for a striped monolayer with fixed $N_{y}=40$, as a function of the aspect ratio $r=N_{x} / N_{y}$. Dots: exact numerical results, Eq. (6). Dashed line: analytical approximation, Eq. (14) with $F(r) \equiv 1$. Full line: asymptotic value (0.09153) of Eq. (14) for $r \rightarrow \infty$. 


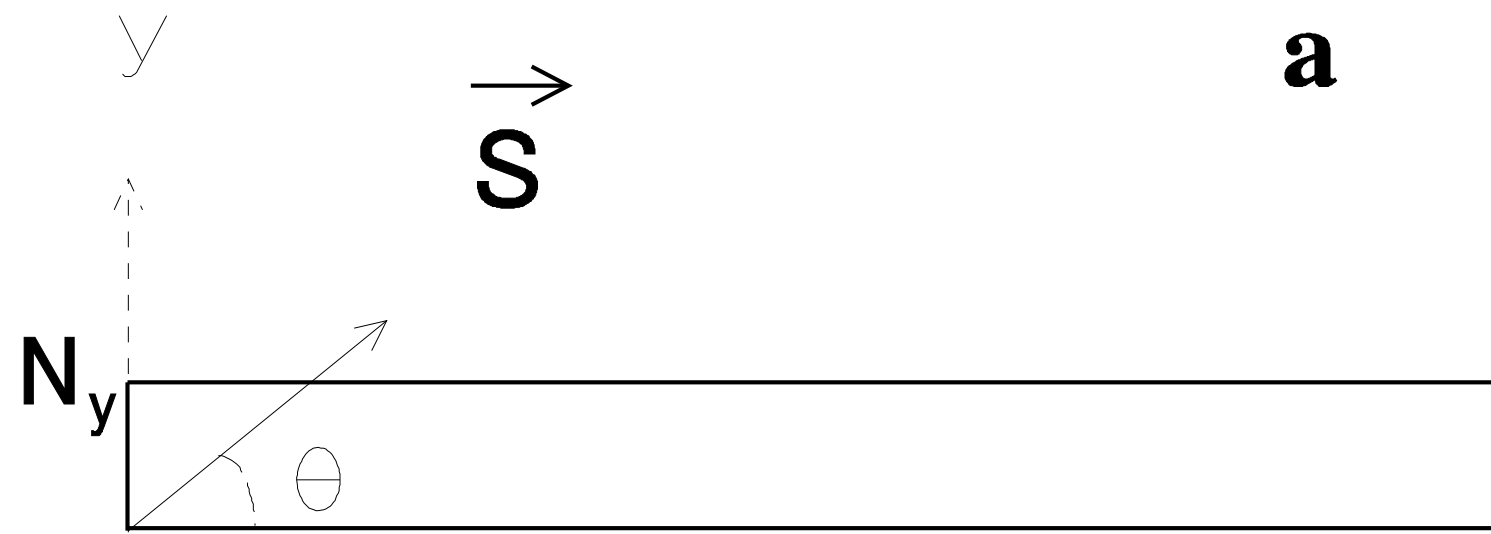

$\mathrm{N}_{\mathrm{x}}$

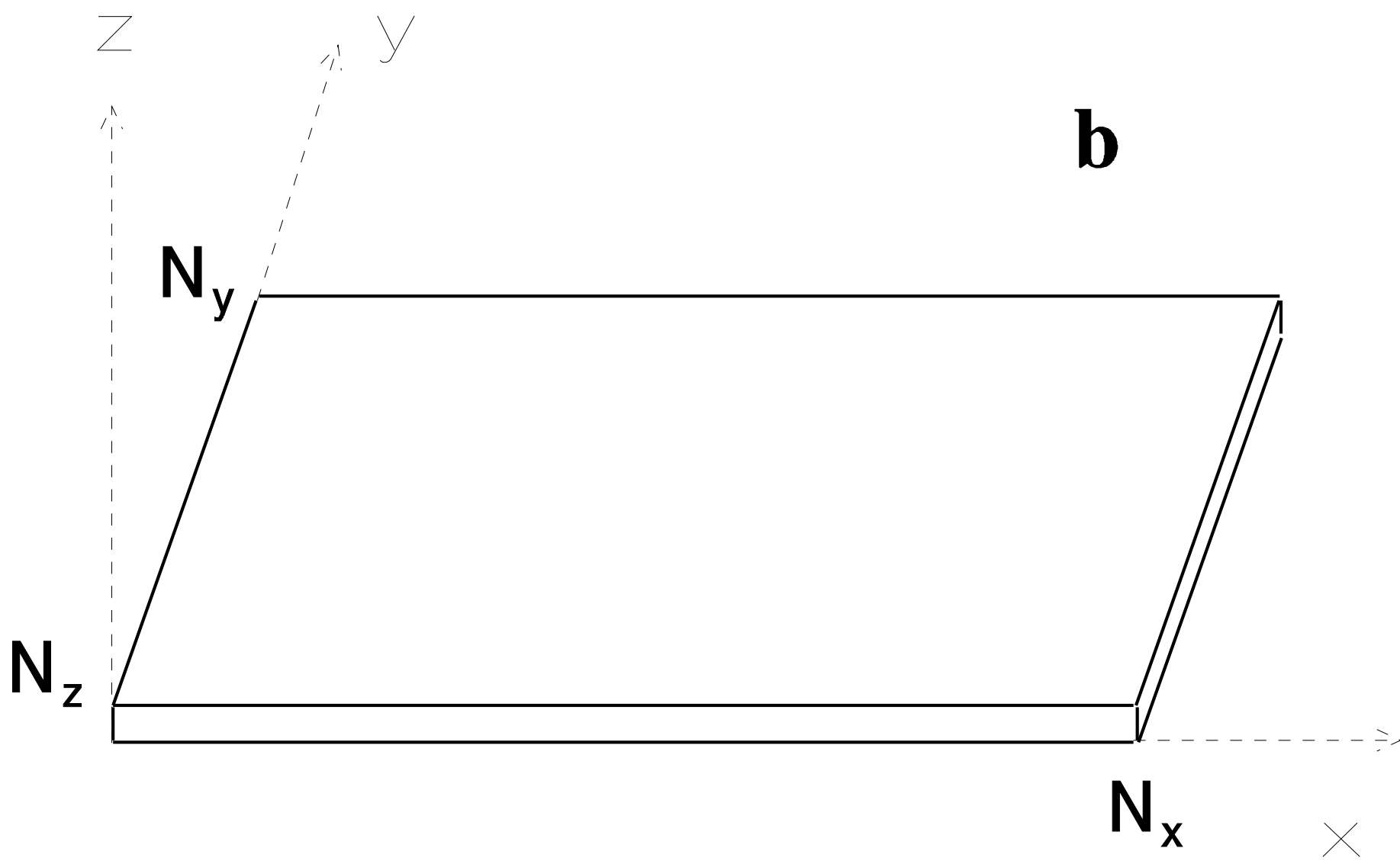




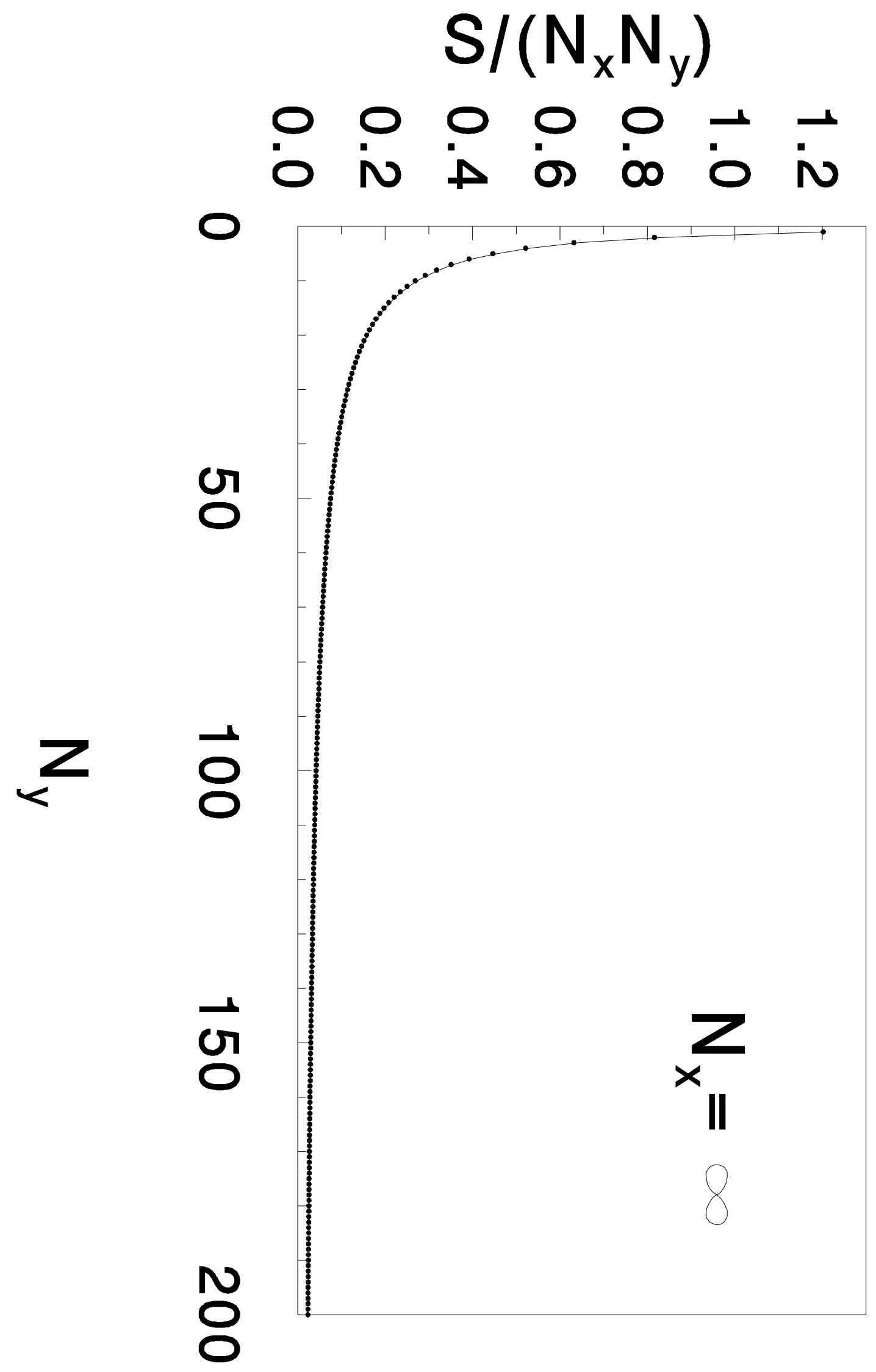


$\mathrm{S} /\left(\mathrm{N}_{\mathrm{x}} \mathrm{N}_{\mathrm{y}}\right)$

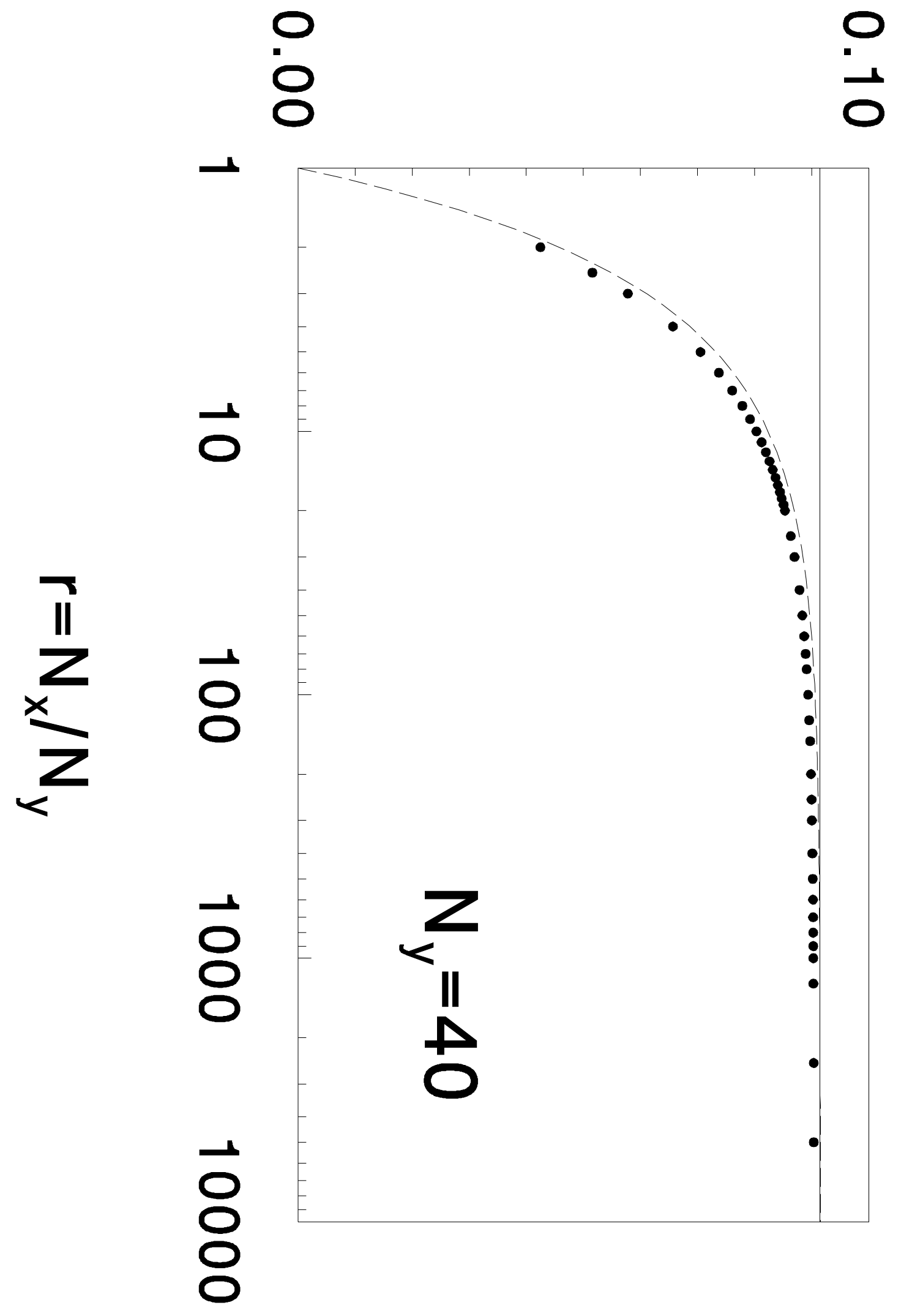

